\title{
Exposure of Carbon Nanotubes Fabricated by Template-Assisted CVD through a Two-Step Method
}

\author{
Yuqin Yao, Quan Xu, Yinjie Cen, Boquan Li, and Jianyu Liang
}

\begin{abstract}
Well aligned carbon nanotubes (CNTs) are highly desirable for various studies and applications. Template-assisted chemical vapor deposition has been widely used. However, post growth surface cleaning to remove the junk carbon on sample surface and expose CNTs still relies on either capital intensive facilities or 'hard to control' processes. In the letter, we report a mechanical polishing method to remove the amorphous carbon layer. Compared to other conventional surface cleaning methods, mechanical polishing is relatively straightforward, much easier to scale up and does not require expensive instruments. Combined with a wet-etching step, highly ordered CNT arrays were reliably obtained. Scanning electron microscopy and atomic force microscopy were employed to investigate the surface morphology and roughness and to optimize the processing conditions.
\end{abstract}

Index Terms-Carbon nanotubes, template-assisted, CVD.

\section{INTRODUCTION}

Since the report by Iijima [1], carbon nanotubes(CNTs) have demonstrated exceptional mechanical, thermal and electric properties due to their unique structures. Hence, CNTs have been vigorously studied as promising candidates for varies applications, including electron field emitter [2], electronic devices [3], [4], bio sensor [5], and drug delivery vessel [6]. For many applications, such as flat panel displays and some sensors, it is essential to have free standing and well aligned CNT arrays with controllable length. In order to provide large area of dense packed and highly ordered CNTs, template assisted method has been developed [7]. During the last decade, several groups have synthesized well-aligned CNTs by using template assisted method for diverse applications [8], [9].

Porous anodized aluminum oxide template (AAO) is a popular template because it can provide uniform, vertical nanopore channels and hexagonal packed structure. It is possible to control the thickness, and pore density and diameter within a certain range by varying several processing parameters, such as choice of electrolyte, applied DC voltage and anodization duration [10]. This controllability is critical to control the dimension (length and diameter) of the final CNTs. Furthermore, template assisted CCVD method can be scaled-up for manufacturing. However, in this method, after the growth of CNTs, there's always a layer of junk carbon on

Manuscript received May 27, 2015; revised July 29, 2015.

Yuqin Yao, Yinjie Cen, Boquan Li, and Jianyu Liang are with Worcester Polytechnic Institute, Worcester, USA (e-mail: yaoyu9115@gmail.com, ycen@wpi.edu,boquanli@wpi.edu,jianyul@wpi.edu).

Quan Xu was with University of North Texas, TX, USA. He is now with China University of Petroleum, Beijing, 102249, China (e-mail: xuquan@cup.edu.cn). the as-made product.

Since for many applications it is essential to remove this layer of junk carbon and controllably expose the highly ordered CNTs, a method to selectively and effectively remove the junk carbon and expose CNTs within the nanopores is highly desirable. Numerous attempts, such as air oxidation and reactive ion etching (RIE) [10]-[15] were studied for this purpose. However, each of these methods has their own pros and cons. Air oxidation is relatively cheap, but it is quite hard to control. It is rather difficult to optimize processing temperature and duration to avoid damaging the CNTs within the nanopores [11], [12]. RIE [10], [13], [15] can reliably and selectively remove the junk carbon layer, but it may take up to 5 hours. Besides, RIE requires rather expensive equipment and sophisticated maintenance, thus is less available outside of large research universities and laboratories.

In this report, a simple, fast and low cost post treatment, which is composed of mechanical polishing and subsequent wet-etching, was demonstrated as an effective method to clean the as-made product surface immediately after CCVD of CNTs in templates and expose the CNTs. Through scanning electron microscopy (SEM) and atomic force microscopy (AFM), it was confirmed that by using small polishing particles a smooth surface with complete removal of junk carbon layer can be obtained through 1 minute of mechanical polishing. Controlled wet etching can be used to further expose the CNTs to desired height.

\section{EXPERIMENTAL}

The CNTs were synthesized by a well-established template assisted CVD method [10], which consists of three steps (Fig. 1): 1). Anodize aluminum sheets to gain nanoporous templates; 2). Grow CNTs via CCVD method; and 3). Post treat the as-made sample to expose the CNTs.

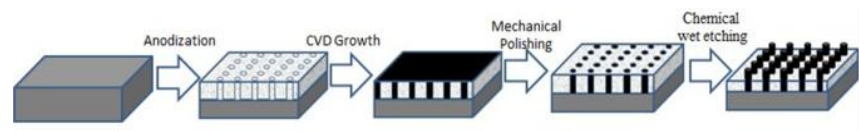

Fig. 1. Schematic process of sample preparation.

First, an aluminum sheet (purity 99.999\%) was anodized via a typical two-step method to generate highly ordered nanoporous templates [10]. Briefly, a first anodization step was carried out in $0.3 \mathrm{M}$ oxalic acid at $40 \mathrm{~V}$ DC and $10{ }^{\circ} \mathrm{C}$ for at least 16 hours. Then a mixture of chromic acid and phosphoric acid was used to remove the AAO thin film on the sheet, followed by the second anodization step conducted under the same conditions for about 12 hours to form highly ordered arrays of hexagonal pores. CCVD was used to grow CNTs inside the nanopores with Cobalt (Co) as catalyst. The 
Co nanoparticles were first electrochemically deposited to the bottom of nanopores. Then the sample was heated up to 600 ${ }^{0} \mathrm{C}$ under CO flow $(80 \mathrm{ml} / \mathrm{s})$ for 2 hours to reduce the Co. A mixture of $\mathrm{C}_{2} \mathrm{H}_{2}(20 \mathrm{ml} / \mathrm{s})$ and $\mathrm{N}_{2}(60 \mathrm{ml} / \mathrm{s})$ was used for CNTs growth for 2 hours. Finally, the sample was annealed in a nitrogen atmosphere at $600{ }^{\circ} \mathrm{C}$ for another 12 hours.

The amorphous carbon layer was removed by mechanical polishing. Polishing particles with difference sizes (Gamma alumina, from Bucheler Inc.) were used in E-plus AC Adjustable Speed Control polishing machine from FHP Inc. Mechanical polishing was carried out for 1 minute for all samples. As a comparison, two other conventional surface cleaning methods, ion milling and air oxidation were also employed to treat identical samples. For a typical ion milling process, the sample was cleaned in $\mathrm{BCl}_{3} 20 \mathrm{sccm}$ and $\mathrm{Ar} 5$ sccm, at Pressure 15 mTorr and Power: $100 \mathrm{~W}$ by a RIE system of Trion Minilock II for 15 minutes [16]. For a typical air oxidation, the sample was heated in the air at $650{ }^{\circ} \mathrm{C}$ for 30 $\min [17]$.

After polishing, the samples were wet etched by $0.01 \mathrm{M}$ $\mathrm{NaOH}$ solution for 20 minutes.

The morphologies of samples were characterized by SEM (JOEL 7000F) and the surface roughness was studied by AFM (MFP3D Bio, Asylum Research) in contact mode.

\section{RESULTS AND DISCUSSION}

After the second anodization, hexagonal nanopores were found to be embedded in the template (Fig. 2 a) ). To extract the diameter distribution of the pores, the SEM images were analyzed with an open source software "Image J [18]". The result shows the size distribution is quite uniform, the mean diameter is $\sim 40 \mathrm{~nm}$ (Fig. 2 b) ).
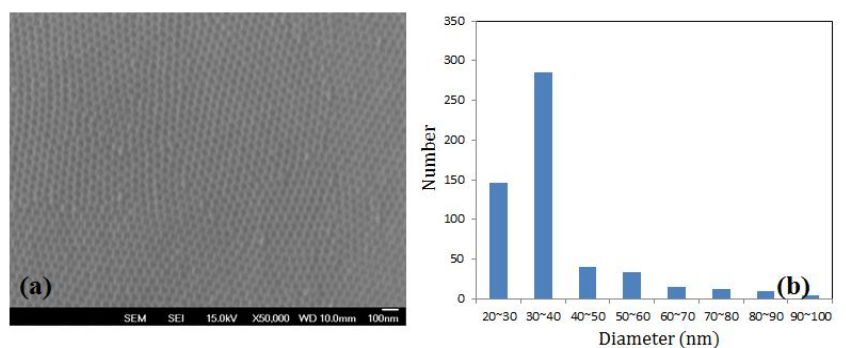

Fig. 2. (a) Top view of the template after the 2nd anodization; (b) Size distribution of AAO template.

After the CCVD synthesis, as shown in Fig. 3 a), a thick amorphous carbon layer was observed on the sample surface, similarly to previous reports and typical for the template-assisted CCVD process [11]. This layer is inert to most etchants and will jeopardize the subsequent wet etching step. From Fig. 3 b), it is obvious that after air oxidation, some of the junk carbon was removed. However, there is still a layer of carbon that blocks the well aligned and ordered CNTs. This result supports the view that the air oxidation method is hard to control.

Similar samples were treated by a reported RIE method [16]. Top view of the sample (Fig. 3 c) ) shows that after RIE, the typical sample surface is flat and the amorphous carbon has been removed. RIE method is more controllable than the air oxidation method. However, the RIE procedure took up a long time ( 5 hours in this experiment), and the equipment is not common on many campuses. Therefore, an alternative method is desired.
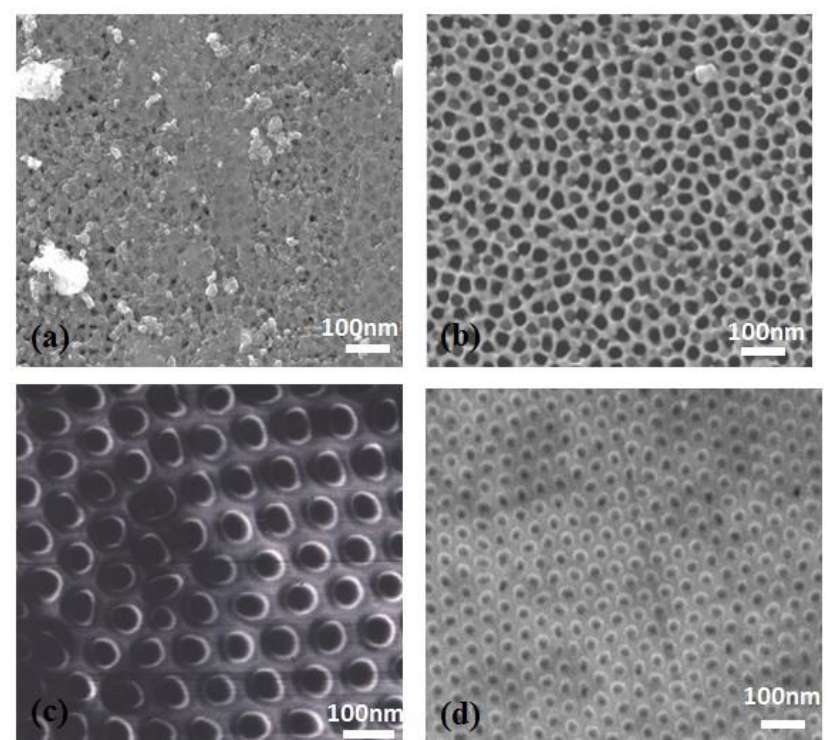

Fig. 3. Top view SEM image of the sample after: (a) CNTs growth; (b) air oxidation; (c) RIE cleaning; (d) mechanical polishing.

The high rigidity of the sample promises the possibility to employ a simple mechanical polishing method to clean the as-made sample surface. Mechanical polishing eliminates the risk of damaging the CNTs. For all mechanical polishing procedures in this study, the duration was 1 minute, and the rotate speed was fixed at $144 \mathrm{rpm}$, three polishing particle sizes were used in this process $(1 \mu \mathrm{m}, 300 \mathrm{~nm}, 50 \mathrm{~nm}$, respectively). Each polished sample was inspected by AFM (Fig. 4(a)-Fig. 4(c) ). The initial scanning area was $10 \times 10 \mu \mathrm{m}$ for all samples. But as shown in the inset of Fig. 4(c), it was hard to tell the details of the sample surface at this scale due to the smoothness of the polished surface. Therefore, for the sample polished by 50nm particles, topography with small scan area $(1 \times 1 \mu \mathrm{m})$ was acquired (Fig. 4(c) ). Table I summarizes the mechanical polishing conditions and the measured surface roughness.

TABLE I: SAMPLE ROUGHNESS AFTER DIFFERENT POLISHING CONDITIONS

\begin{tabular}{|c|c|c|c|}
\hline Duration & $\begin{array}{c}\text { Rotate } \\
\text { speed }\end{array}$ & $\begin{array}{c}\text { Particle } \\
\text { size }\end{array}$ & Roughness(RMS) \\
\hline $1 \mathrm{~min}$ & $144 \mathrm{rpm}$ & $50 \mathrm{~nm}$ & $2.1 \mathrm{~nm}$ \\
\hline $1 \mathrm{~min}$ & $144 \mathrm{rpm}$ & $300 \mathrm{~nm}$ & $3.4 \mathrm{~nm}$ \\
\hline $1 \mathrm{~min}$ & $144 \mathrm{rpm}$ & $1 \mu \mathrm{m}$ & $16.4 \mathrm{~nm}$ \\
\hline
\end{tabular}

Fig. 3(d) is the SEM top view image of a typical sample surface that was polished by $50 \mathrm{~nm}$ particles for $1 \mathrm{~min}$. The result was comparable to the surface cleaning by RIE (Fig. 3(c) ). It is obvious that the amorphous carbon has been removed and CNTs were visible in the template matrix after 1 min mechanical polishing. SEM and AFM results provide the evidence proving that mechanical polishing using particle size of $50 \mathrm{~nm}$ can effectively clean the samples made by template assisted CCVD.

Fig. 5(a) is the top view image of the sample after etching 
with $0.01 \mathrm{M} \mathrm{NaOH}$ for $20 \mathrm{~min}$, the CNTs were successfully further exposed (Fig. 5(a)) and the matrix remains flat. To measure the exposed length of CNTs accurately, AFM topographic measurements (one typical test shown in Fig. 5(b) and Fig. 5 (c) ) confirmed that the CNTs in the samples treated by this post-growth process were exposed rather uniformly. The height is about $50 \mathrm{~nm}$ as identified by Fig. 5(d).

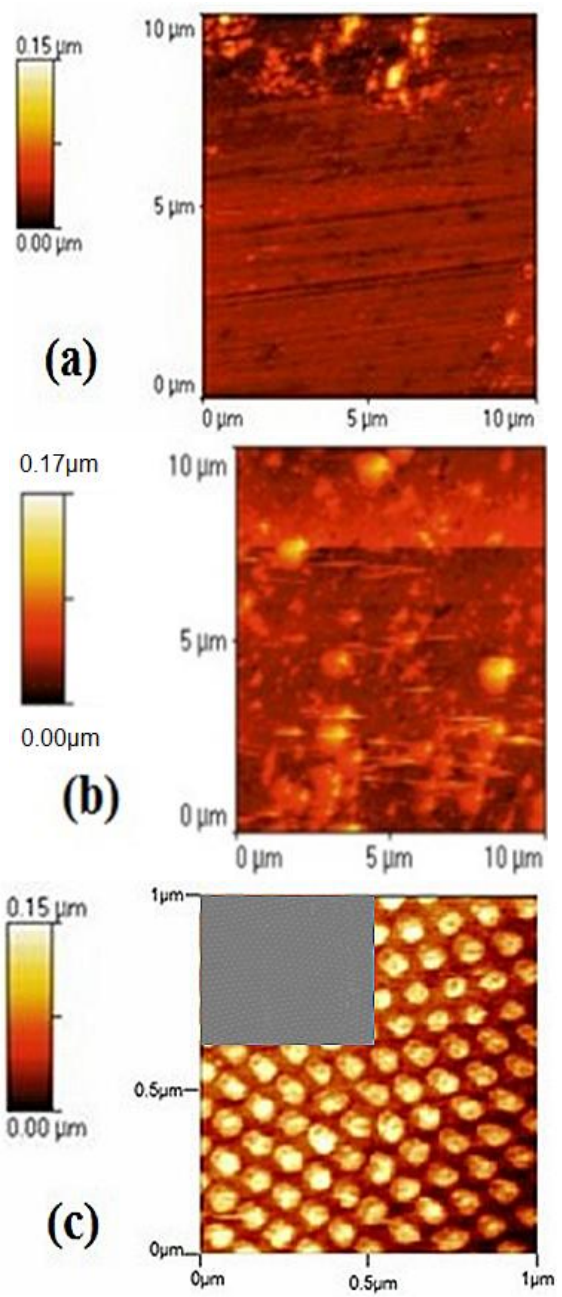

Fig. 4. AFM topograhies after polished by particles of (a) $1 \mu \mathrm{m}$ (b)300nm (c) $50 \mathrm{~nm}$ in diameter (the inset shows AFM topography of $10 \times 10 \mu \mathrm{m}$ ).

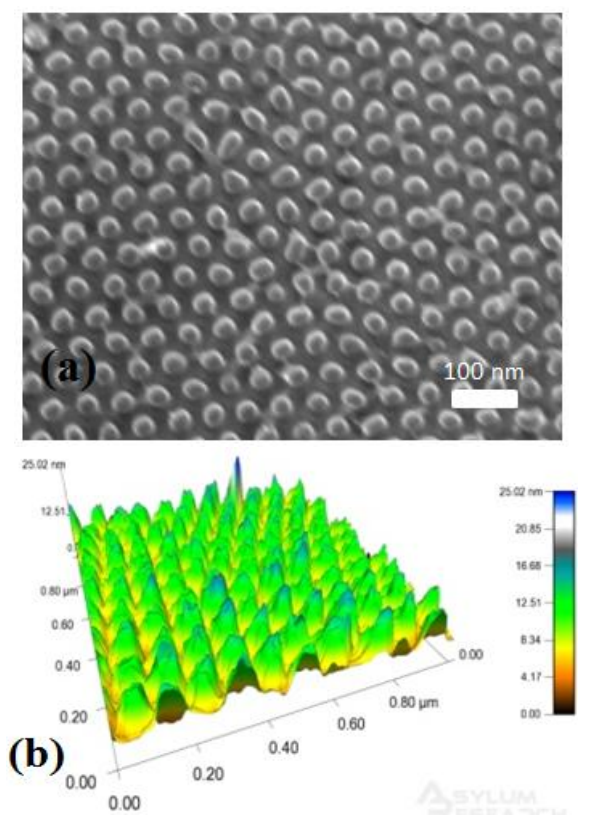

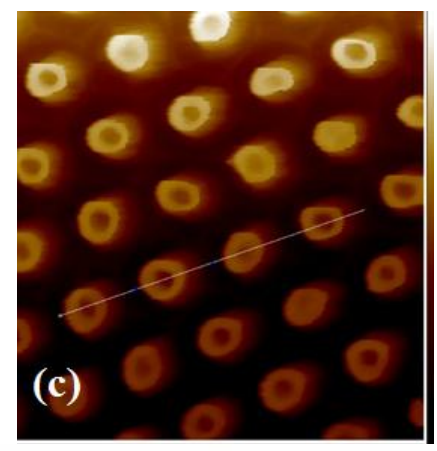

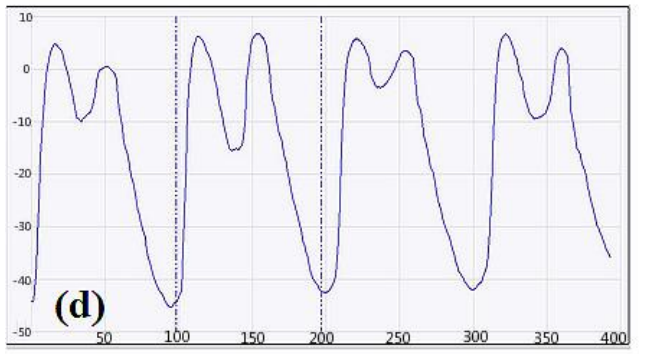

Fig. 5. (a) SEM top view image; (b) 3-D image of the sample after post-treatment; (c) AFM topography of the sample; (d) cross sectional profile of the sample.

\section{CONCLUSIONS}

In conclusion, the CCVD process based on template-assisted method is a convenient way to synthesize highly ordered CNTs in large scale. AAO templates made via two-step anodization method can serve as a platform to provide uniform nanopores for the synthesis of well-aligned carbon nanotubes. Mechanical polishing combined with wet-etching method can be considered as a low cost and effective alternative method to clean the surface and expose CNTs, it is relatively simple, straightforward, controllable, and useful for the future applications, such as interfacial mechanical test, electric applications, etc

\section{REFERENCES}

[1] S. Iijima, "Helical microtubules of graphitic carbon," Nature, vol. 354, issue 6348, pp. 56-58, 1991.

[2] Y. Saito and S. Uemura, "Field emission from carbon nanotubes and its application to electron sources," Carbon, vol. 38, no. 2, pp. 169-182, 2000.

[3] J. Liu et al., "Controlled deposition of individual single-walled carbon nanotubes on chemically functionalized templates," Chemical Physics Letters, vol. 303, issue 1-2, pp. 125-129, 1999.

[4] C. Kocabas, M. Shim, and J. A. Rogers, "Spatially selective guided growth of high-coverage arrays and random networks of single-walled carbon nanotubes and their integration into electronic devices," Journal of the American Chemical Society, vol. 128, no. 14, pp. 4540-4541, 2006.

[5] K. Besteman et al., "Enzyme-coated carbon nanotubes as single-molecule biosensors," Nano Letters, vol. 3, no. 6, pp. 727-730, 2003.

[6] J. Chen et al., "Functionalized single-walled carbon nanotubes as rationally designed vehicles for tumor-targeted drug delivery," Journal of the American Chemical Society, vol. 130, no. 49, pp. 16778-16785, 2008.

[7] P.-L. Chen et al., "Anodic aluminum oxide template assisted growth of vertically aligned carbon nanotube arrays by ECR-CVD," Diamond and Related Materials, vol. 13, no. 11, pp. 1949-1953, 2004.

[8] S. Wen et al., EDLC characteristics with high specific capacitance of the CNT electrodes grown on nanoporous alumina templates," Current Applied Physics, vol. 6, no. 6, pp. 1012-1015, 2006.

[9] S.-H. Jeong et al., "Template-based carbon nanotubes and their application to a field emitter," Applied Physics Letters, vol. 78, no. 14, pp. 2052-2054, 2001. 
[10] J. Liang, H. Chik, and J. Xu, "Nonlithographic fabrication of lateral superlattices for nanometric electromagnetic-optic applications," IEEE Journal of Selected Topics in Quantum Electronics, vol. 8, no. 5, pp. 998-1008, 2002.

[11] X. Chen et al., "Non-destructive purification of multi-walled carbon nanotubes produced by catalyzed CVD," Materials Letters, vol. 57, no. 3, pp. 734-738, 2002.

[12] S. J. Kang et al., "High-performance electronics using dense, perfectly aligned arrays of single-walled carbon nanotubes," Nature Nanotechnology, vol. 2, no. 4, pp. 230-236, 2007.

[13] Z. Ren et al., "Synthesis of large arrays of well-aligned carbon nanotubes on glass," Science, vol. 282, no. 5391, pp. 1105-1107, 1998.

[14] S. Huang and L. Dai, "Plasma etching for purification and controlled opening of aligned carbon nanotubes," The Journal of Physical Chemistry B, vol. 106, no. 14, pp. 3543-3545, 2002.

[15] M. Chhowalla et al., "Growth process conditions of vertically aligned carbon nanotubes using plasma enhanced chemical vapor deposition," Journal of Applied Physics, vol. 90, no. 10, pp. 5308-5317, 2001.

[16] A. Yin, H. Chik, and J. Xu, "Postgrowth processing of carbon nanotube arrays-enabling new functionalities and applications," IEEE Transactions on Nanotechnology, vol. 3, no. 1, pp. 147-151, 2004.

[17] M. Shiraishi and M. Ata, "Work function of carbon nanotubes," Carbon, vol. 39, no. 12, pp. 1913-1917, 2001.

[18] Image. [Online]. Available: http://imagej.nih.gov/ij/

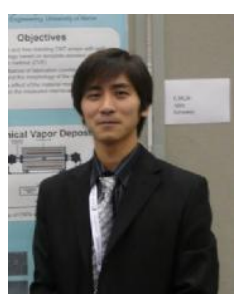

Yuqin Yao received his B.S. and M.S. degrees from the Department of Chemical Engineering, Nanjing Tech University, China and his Ph.D. degree from the Department of Materials Science and Engineering, Worcester Polytechnic Institute, USA. His research interests are focused on synthesis of carbonaceous nanomaterials for mechanical and energetic applications.

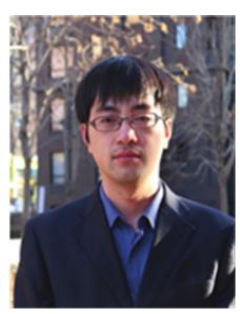

Quan Xu received his Ph.D. degree from the Department of Materials Science and Engineering University of North Texas in 2013, with Prof. Zhenhai Xia. He joined the Faculty of the Institute of New Energy, China University of Petroleum, Beijing in 2014 and now is an associate professor. His research interests are focused on the development of membrane catalysis technology, and synthesis of nanomaterials for environmental applications as well as biotechnology.

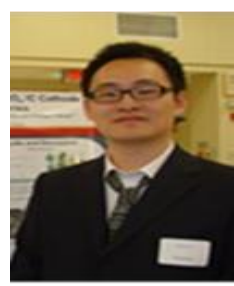

Yinjie Cen received his bachelor's degree from the Department of Materials Science and Engineering at Central South University in 2010. He is now a Ph.D. candidate at Worcester Polytechnic Institute (WPI) and joined Prof. Liang's group in 2012. His research interests are focused on developing and studying the synthesis and characterization of new electrode materials for $\mathrm{Li}$-ion batteries.

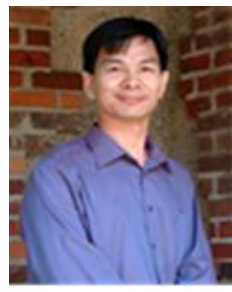

Boquan $\mathbf{L i}$ received his Ph.D. degree from the Center for Fundamental Physics, University of Science and Technology of China. He joined the materials science and engineering program, Worcester Polytechnic Institute in 2005. His research interests are focused on the development of advanced $\mathrm{x}$-ray and electron microscopic methods for structural characterization of additive manufactured materials, nanostructured materials and thin films.

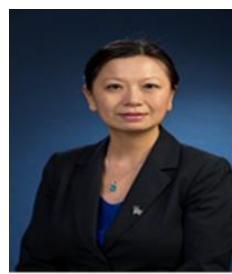

Jianyu Liang is an associate professor of mechanical engineering and chemical engineering. She has also served as a guest researcher at Army Research Laboratory during her sabbatical leave in 2013-2014 academic years. Her current research work includes nanomaterials for batteries and fuel cells, cold spray of nanostructured metallic materials, and green manufacturing of construction materials from industrial wastes and by products. She has authored over 60 articles in the areas of nanotechnology, materials science, and electrochemistry and gave over 100 invited talks, seminars, presentations and speeches. Her current work is supported by ARL and Department of Education. 